\title{
MORPHOLOGY OF THE PULMONARY AND AORTIC ROOTS WITH REGARD TO THE PULMONARY AUTOGRAFT PROCEDURE
}

Raymond B. Hokken, MD Margot M. Bartelings, MD, $\mathrm{PhD}^{\mathrm{b}}$ Ad J. J. C. Bogers, MD, PhD Adriana C. Gittenberger-de Groot, $\mathrm{PhD}^{\mathrm{b}}$
Aortic root replacement with the pulmonary autograft warrants a thorough histologic comparison of the morphologic characteristics of the pulmonary and aortic roots. For this purpose nine normal heart specimens ( 7 neonatal and 2 adult hearts) were studied. Histologic study confirmed the collagenous anulus in both roots to be a complex circular-shaped structure, intricately interposed between the elastic lamellae of the arterial wall and the ventricular structures of the heart. In the sinus the elastic lamellae of the arterial wall continue along the luminal side with collagen being situated at the outside. At the interleaflet triangle this relation is reversed. Surprisingly, islets of elastic fibers were found in the otherwise completely collagenous interleaflet triangles. The amount of elastic lamella distal to the commissures was in both arteries higher than that in the middle of the sinuses, with a preponderance in the aorta as compared with the pulmonary trunk. The pulmonary root anulus proximally inserts into the relatively thin right ventricular myocardium, whereas the aortic root anulus inserts into the thick left ventricular myocardium and several fibrous structures. The pulmonary root is hardly supported by the right ventricular myocardium, whereas the aortic root is supported by its wedged position between the left and right atrioventricular anuli and the bulging thick left ventricular myocardium. When the pulmonary autograft is used for aortic root replacement it should be inserted as proximally as possible to get the support of the fibrous structures of the left ventricular outflow tract and the surrounding ventricular and atrial myocardium. ( $J$ Thorac Cardiovasc Surg 1997;113:453-61)
$\mathrm{M}$ orphologic descriptions of the aortic and pulmonary valve and root had already been published by the first half of this century. ${ }^{1,2}$ More recent studies describe the morphology of the aortic valve and root in relation to function ${ }^{3}$ and in relation to congenital malformations., ${ }^{4,5}$ With the use of the pulmonary autograft for aortic root replacement, a

From the Department of Cardiopulmonary Surgery ${ }^{\text {a }}$ of the University Hospital Dijkzigt, Rotterdam, and the Department of Anatomy and Embryology ${ }^{\mathrm{b}}$ of Leiden University, Leiden, The Netherlands.

Supported in part by a grant from the Netherlands Heart Foundation (NHS No. 89.237).

Received for publication July 2, 1996; revisions requested August 27, 1996; revisions received Sept. 26, 1996; accepted for publication Sept. 30, 1996.

Address for reprints: Margot M. Bartelings, MD, PhD, Department of Anatomy and Embryology, Leiden University, P.O. Box 9602, 2300 RC Leiden, The Netherlands.

Copyright (C) 1997 by Mosby-Year Book, Inc.

$0022-5223 / 97 \$ 5.00+0 \quad \mathbf{1 2 / 1 / 7 8 3 8 0}$ method developed by Ross ${ }^{6}$ in 1986, the question with regard to the suitability of the pulmonary valve and root for use in the aortic position is relevant. The clinical results of the pulmonary autograft implanted in the aorta with the subcoronary technique $^{7}$ are good. ${ }^{8}$ However, when the complete aortic root is replaced by the pulmonary root, not only the pulmonary valves but also the pulmonary wall are exposed to systemic pressures. Although the short-term results with this technique are satisfactory with regard to the clinical performance, ${ }^{9-11}$ some surgeons advise wrapping the pulmonary autograft to avoid dilation. ${ }^{7,12}$ In this regard the pathophysiologic nature of native aortic regurgitation caused by aortic root dilation ${ }^{13,14}$ may also be applicable to the pulmonary autograft in the aortic position.

Therefore a microscopic morphologic study of the components of the pulmonary and aortic roots and their proximal insertions with a description of the surrounding structures seems appropriate and may 


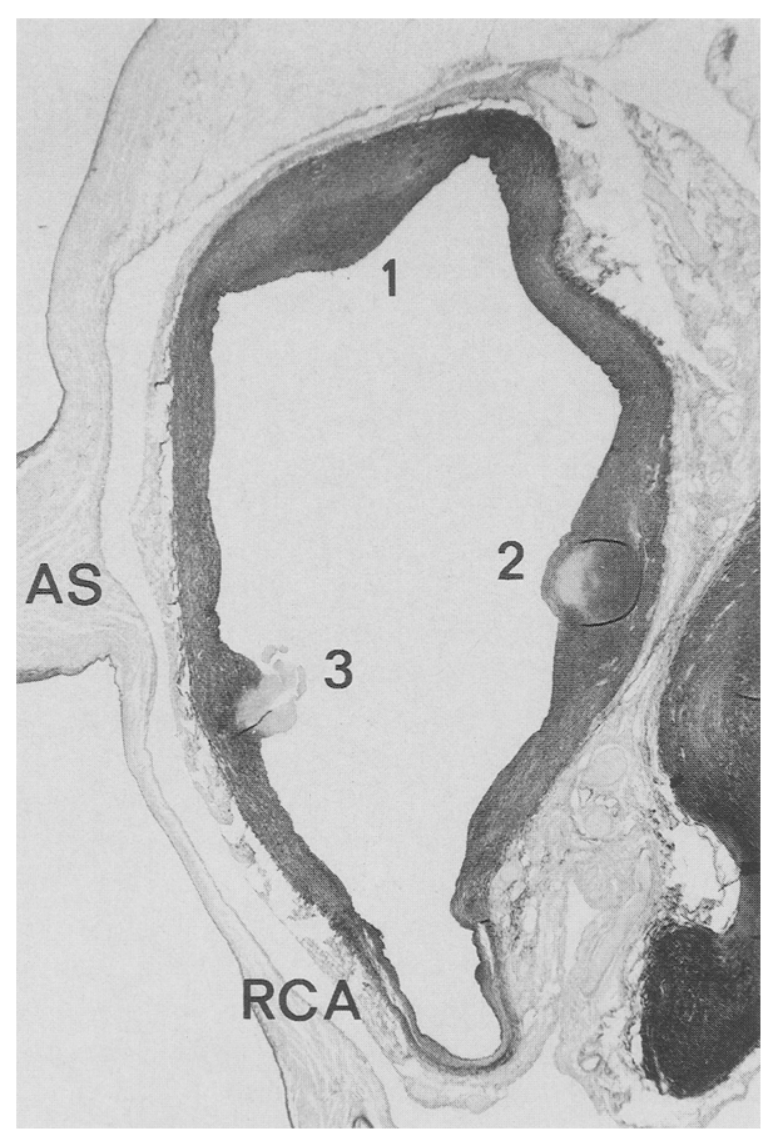

Fig. 1. Section of the distal part of the aortic root. 1 , Protrusion of the elastic (darkly stained) vessel wall, slightly above a commissure; 2, collagenous (lightly stained) area surrounded by elastic lamellae, constituting the most distal extension of the commissure; 3 , the commissure itself, where the two valve leaffets meet. $A S$, Atrial septum; $R C A$, right coronary artery.

give insight into the differences between the pulmonary and aortic roots in general and the consequences of implantation of the pulmonary autograft in the aortic position.

\section{Material and methods}

Material. Nine normal heart specimens were studied. Seven hearts of children who died perinatally at a mean gestational age of 38 weeks (range 34 to 40 weeks) were obtained from the Leiden Collection of heart-lung specimens. Two adult hearts ( 42 and 47 years old) from the Department of Pathology of the University Hospital Rotterdam were included to investigate possible age-related differences.

Methods. The specimens were cut in serial sections approximately perpendicular to the aortic axis with a thickness of $10 \mu \mathrm{m}$. Four consecutive sections of the entire specimen were put together on one slide. The slides were stained alternately with azan, resorcin-fuchsin, hematoxylin-eosin, and modified van Gieson's stains. Because the pulmonary axis is not parallel to the aortic axis, the plane of sectioning of the pulmonary trunk was not transverse. With use of these sections three-dimensional reconstructions were made to clarify and describe the relationships between the elastic and collagenous components of the pulmonary and aortic roots and of their insertions in the myocardium.

Elastic lamellae in the wall of the pulmonary trunk and aorta were counted in each specimen at six different places: in the middle of each of the three sinus walls and $2 \mathrm{~mm}$ distal to each of the commissures.

Terms were defined as follows.

Pulmonary root. The pulmonary root was defined as the first part of the pulmonary trunk, from the insertion of the pulmonary anulus in the right ventricular myocardium, including the semilunar valve leaflets, the wall of the sinuses, the interleaflet triangles, the commissures, and the sinotubular junction.

Aortic root. The aortic root is the first part of the aorta, from the insertion of the aortic anulus in the left ventricular myocardium and the continuation in proximal fibrous structures, including the semilunar valve leaflets, the wall of the sinuses of Valsalva (with the coronary orifices), the interleaflet triangles, the commissures, and the sinotubular junction.

Sinuses. The sinuses include pockets or cavities of the pulmonary and aortic roots between the arterial wall and the arterial side of the semilunar valve leaflets. The sinuses of the aorta are named according to the coronary arteries with their ostia (left and right coronary sinus), and the sinus without a coronary ostium is the noncoronary sinus. The pulmonary sinuses are named by their relationship to the aortic sinuses: left facing, right facing, and nonfacing sinuses. ${ }^{15}$

Sinotubular junction. The sinotubluar junction was defined as the borderline between the more distal arterial wall and the thinner arterial wall of the sinuses.

Commissures. The commissures are the sites at the arterial wall where two valve leaflets meet.

Interleaflet triangle. The triangular part of the arterial wall in between two sinuses with its base on the ventricular myocardium and extending up to the commissures is the interleaflet triangle., ${ }^{4,5}$

Anulus. The anulus is the fibrous structure in the arterial root to which the semilunar valve leaflets are attached.

Proximal fibrous structures. Proximal fibrous structures are fibrous structures proximal to the aortic anulus.

Surrounding structures. Peripheral structures that lie adjacent to the aortic or pulmonary root are surrounding structures.

Statistical analysis. The mean values of the elastic lamella counts at both levels in the pulmonary trunk and aorta were calculated. A $t$ test was used to evaluate the differences between both levels of pulmonary trunk and aorta and between both arteries at the same level.

\section{Results}

Pulmonary root. The distal part of the pulmonary trunk consists largely of elastic lamellae arranged in 


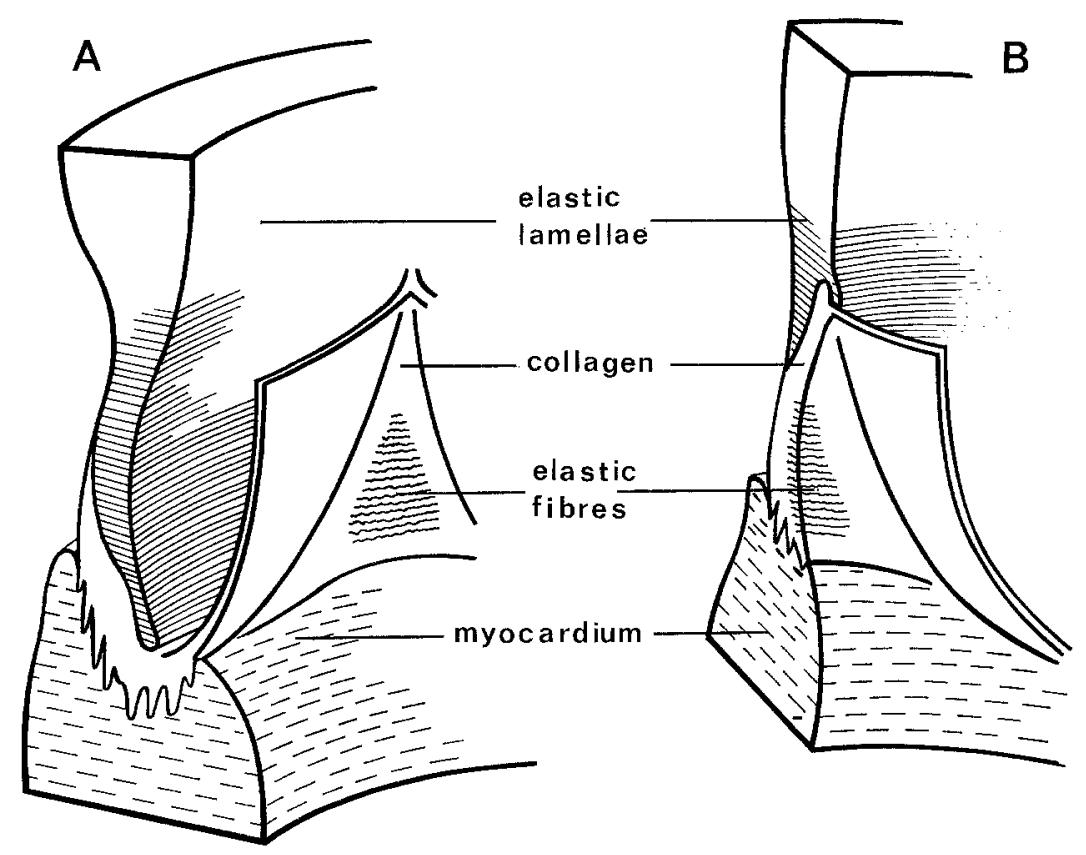

Fig. 2. Schematic drawings of the arterial root after three-dimensional reconstruction showing longitudinal sections through (A) the middle of a sinus wall and $(\mathbf{B})$ the middle of a commissure and interleaflet triangle.

a concentric fashion. Collagen and smooth muscle cells are visible between its layers. Going upstream the pulmonary wall shows three protrusions at the luminal side. Further toward the heart, collagenous condensations are found in the center of these protrusions, constituting the most distal extension of the commissures. Slightly more proximally, these collagenous condensations bulge into the arterial lumen, and here the valve leaflets originate (Fig. 1 and Fig. 2, $A$ and $B$ ).

The collagenous tissue of the commissures is continuous with the collagenous tissue of the interleaflet triangles situated between the diverging valve leaflets. The apical one third of the triangle, like the commissures, consists of collagenous tissue on the inside and elastic lamellae on the outside. Going upstream, the thickness of the outer elastic wall gradually decreases until the entire thickness of the wall is formed by collagen (Fig. 2, $A$ and $B$, and Fig. 3). In the middle of the interleaffet triangle an isolated condensation of elastic fibers is present at the luminal side, which because it is more obvious in the aortic root will be further described in that section.

Although it has the same components as the interleaflet triangles, the wall of the adjacent sinuses has a different design. The distal part of the sinus wall consists of concentric elastic lamellae, comparable to the more distal vessel wall. Going upstream, the number of elastic lamellae decreases and the amount of collagen between the elastic lamellae increases. In the proximal half of the sinus wall the layer of elastic lamellae is restricted to the luminal side. The outer side is formed by a collagenous layer, which is continuous with the interleaflet triangles (Fig. 2, $A$ and $B$, and Fig. 3). In contrast to the elastic component in the interleaflet triangle where it becomes more and more limited to the outer side of the wall, in the sinus wall the elastic component is restricted to the luminal side, until it disappears at the base of the sinus. Here collagenous tissue constitutes the entire thickness of the wall (Fig. 2, $A$ and $B$, and Fig. 3). At the base of the interleaflet triangles and the sinuses many fingerlike protrusions of the collagenous tissue extend into the underlying myocardium, providing an extensive anchorage of the base of the arterial root. The larger part of the pulmonary anulus inserts into the free wall of the right ventricular myocardium. The interleaflet triangle between the left and right pulmonary sinuses, with parts of the right and left pulmonary 


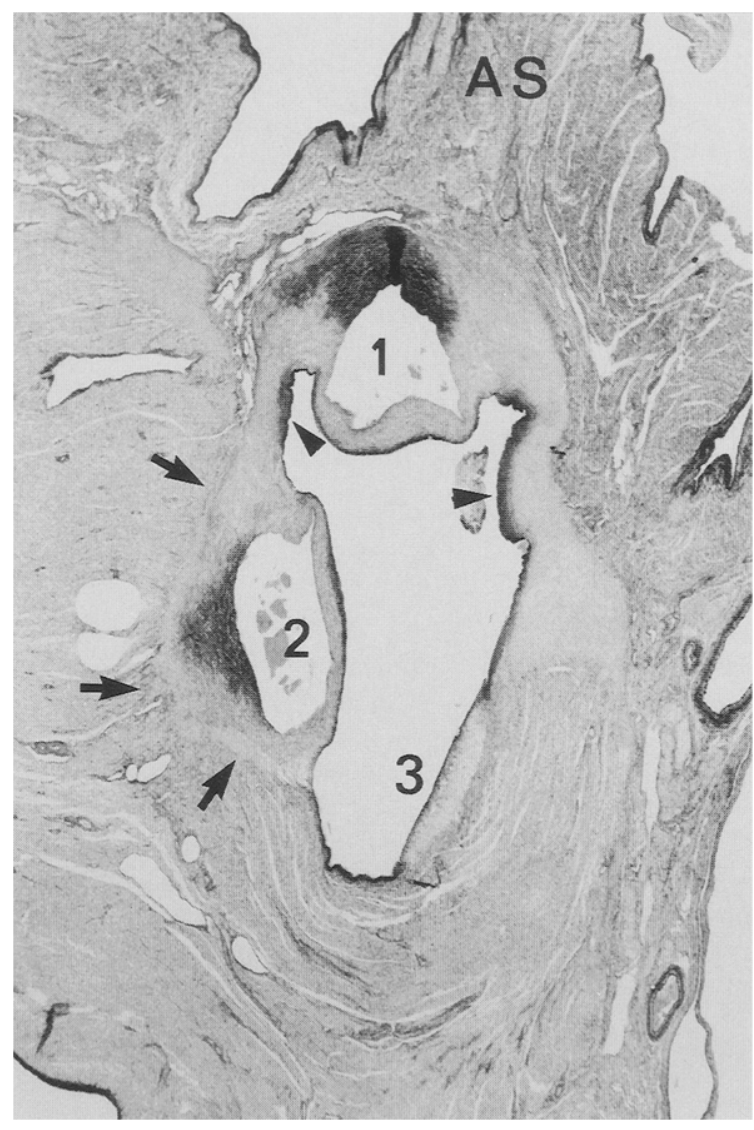

Fig. 3. Section through the aortic root at a more proximal level than that in Fig. 1. The arrows indicate part of the borderline between the lightly stained collagen and the slightly darker stained myocardium. The elastic lamellae are darkly stained. 1, Noncoronary sinus with elastic lamellac makes up the entire thickness of the sinus wall; 2, slightly more proximally, the right coronary sinus shows elastic lamellae on the luminal side and collagenous tissue inserting into the myocardium on the outer side; 3 , more proximally, at the base of the left coronary sinus, there is only collagenous tissue. The arrowheads indicate the elastic fibers at the luminal side of the interleaflet triangles. $A S$, Atrial septum.

sinuses, inserts in the septal part of the right ventricular myocardium (Fig. 4, $A$ ).

The valve leaflets originate from the intricately shaped, circular, collagenous structure that extends from the commissures, where it is wedged into the elastic vessel wall, to the base of the sinuses and the interleaflet triangles. It should be noted, however, that the border of the underlying right ventricular myocardium is curved, thus the base of the inter- leaflet triangles is situated more cranially than the base of the sinuses.

The pulmonary root has no surrounding structures except for the adjacent aorta. In some cases $(n=2)$ a collagenous connection was present between the aortic and pulmonary roots. This collagenous connection extended from the interleaflet triangle between the right and left pulmonary sinuses to the interleaflet triangle between the right and left coronary sinuses of the aortic root (Fig. 5). The right ventricular myocardium, the septal part more than the thin free wall, slightly bulges on the outside. Loose connective tissue is situated in between the pulmonary root and the myocardium.

Aortic root. Although the general principle of a collagenous structure that is intricately interposed between a largely elastic vessel wall and ventricular structures, as described in the section on the pulmonary root, is also applicable to the aortic root, there are major and minor differences between the roots. One of the minor differences is that the sinus walls and the interleaflet triangles of the aorta appear to be thicker. As mentioned previously, the isolated condensations of elastic fibers in the collagenous interleaflet triangles are more pronounced. These elastic islets are not continuous with the elastic lamellae of the arterial wall and they are situated at the luminal side of the triangle (Figs. 3 and 6). With the decreasing thickness of the interleaflet triangle, toward the heart, the number of elastic fibers decreases until they are absent at the base of the triangle. The proximal part of the right coronary sinus was continuous with a discrete accumulation of collagen in three specimens; this accumulation of collagen was in direct continuity with the membranous septum in one specimen, whereas in the others this was not the case (Fig. 7).

With regard to the anchorage of the roots in the ventricular structures major differences exist between the aortic and pulmonary roots. Slightly less than half of the aortic root is inserted in the myocardium of the left ventricle. This applies to half of the left coronary sinus wall, the interleaflet triangle between left and right coronary sinuses, and the right coronary sinus wall (Fig. $4, B$, and Table I). Similar to the pulmonary root, the base of the collagenous part of the aortic root in this area has fingerlike protrusions that extend into the underlying myocardium. This myocardium is, however, much thicker than that of the right ventricle, which provides a broad area of connection between the collagenous protrusions and the myocardium. 

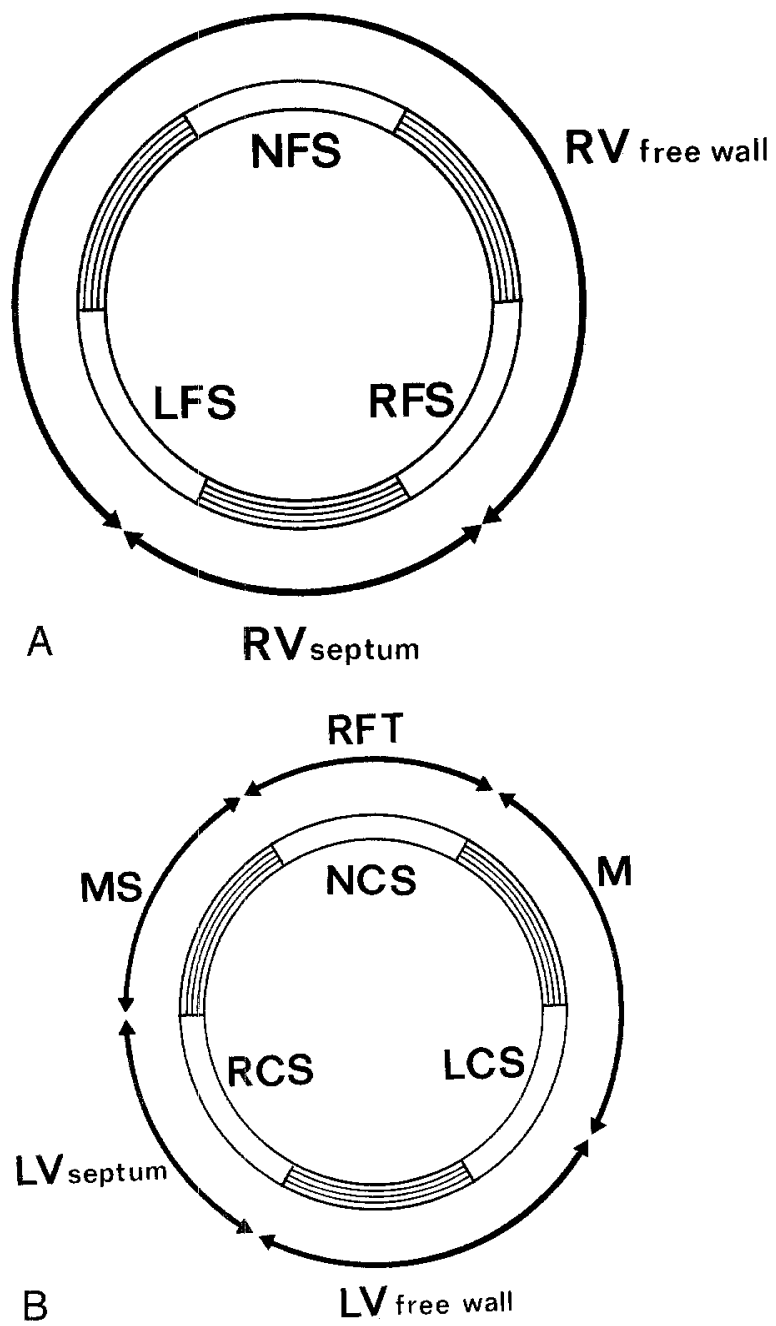

Fig. 4. A, Schematic representation of the pulmonary root related to the site of insertion into the myocardium. $R V$, Right ventricle; RFS, right facing sinus; $L F S$, left facing sinus; NFS, nonfacing sinus. B, Schematic representation of the aortic root related to the site of insertion into the myocardium and fibrous structures. The left fibrous trigone is situated between the anterior mitral valve leaflet and the left ventricular free wall. $L V$, Left ventricle; $R C S$, right coronary sinus; $L C S$, left coronary sinus; $N C S$, noncoronary sinus; $M S$, membranous septum; $R F T$, right fibrous trigone; $M$, anterior mitral valve leaflet.

More than half of the aortic root circumference does not insert in myocardium but continues in proximal fibrous structures (Fig. 4, $B$, Table I). The interleaflet triangle between the right coronary and noncoronary sinuses proximally continues in the membranous septum, which separates the left ventricle from the right ventricle and right atrium (Fig.

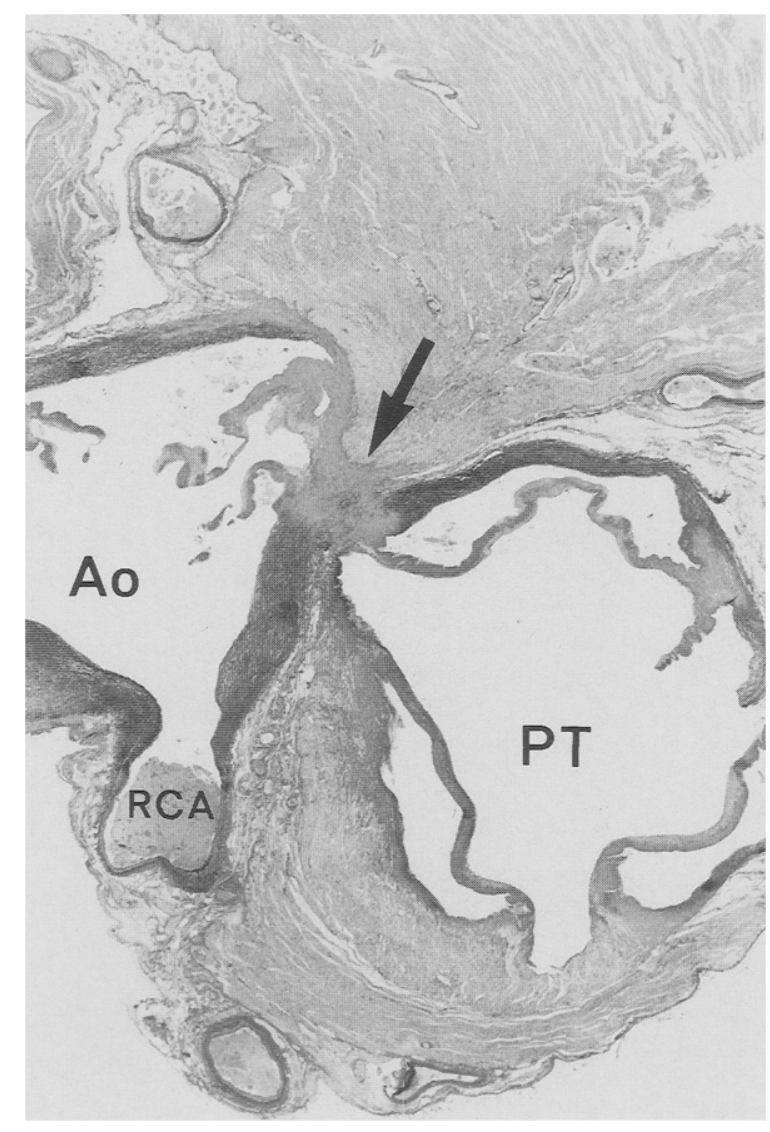

Fig. 5. Section of the pulmonary and aortic roots. The arrow indicates the conus tendon between both roots. $A O$, Aorta; $P T$, pulmonary trunk; $R C A$, right coronary artery.

7). Proximally, the right atrial part of this membranous septum inserts into the atrioventricular (muscular) septum, separating the right atrium and left ventricle. This part contains the atrioventricular node. The right ventricular part of the membranous septum inserts proximally into the ventricular septum myocardium, which contains conducting tissue (bundle of His). The left fibrous trigone is situated at the proximal part of the left sinus. At one side it is continuous with the interleaflet triangle between the left and noncoronary sinuses, which proximally continues in the anterior mitral valve leaflet. The other side inserts into the left ventricular myocardium (Fig. 4, B, Table I). The right fibrous trigone, presenting as a huge accumulation of collagen, is located adjacent to the base of the noncoronary sinus (Fig. 7). Both the anterior mitral valve leaflet (with the left fibrous trigone) and the membranous septum are continuous with this structure (Fig. 4, B). 


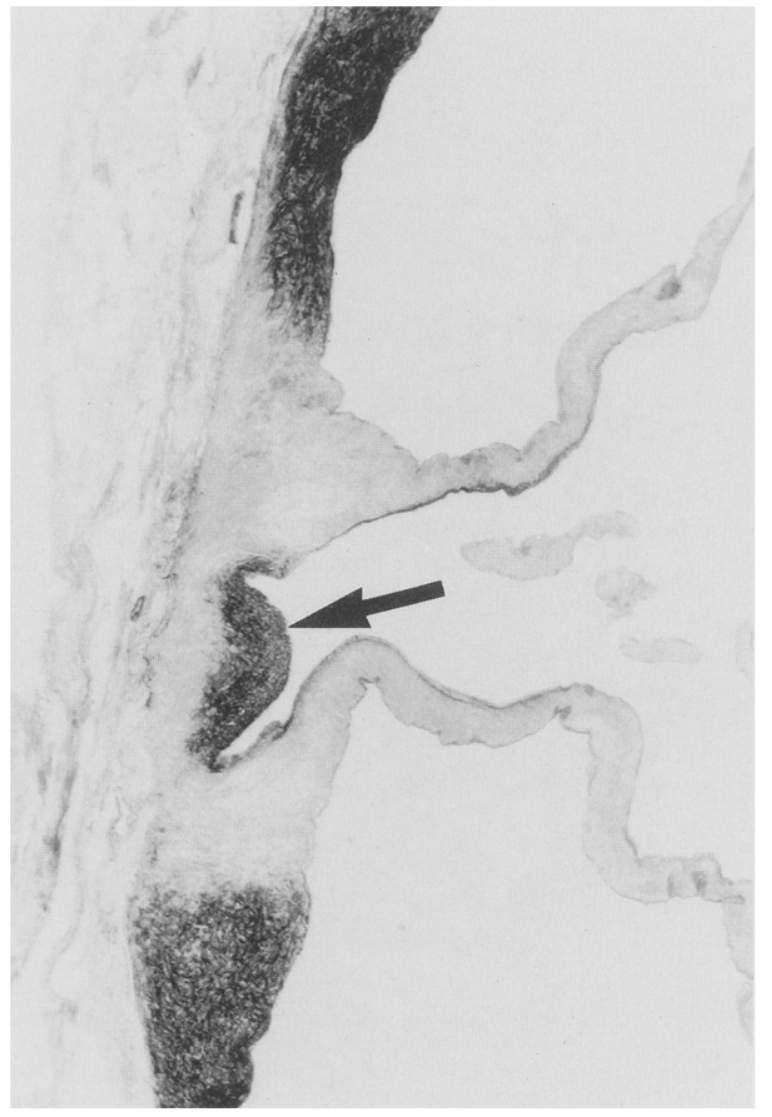

Fig. 6. Detail showing part of the isolated condensation of elastic fibers (arrow) in the interleaflet triangle, reconstructed in Fig. 2.

Proximally the right fibrous trigone inserts into the left ventricular myocardium.

The aortic root has more surrounding structures than the pulmonary root. The thick left ventricular myocardium and septal myocardium bulge on the outside, forming a collar around the proximal part of the aortic root. Loose connective tissue is situated between the myocardium and the aortic root. The anuli of the right and left atrioventricular valves are continuous with the membranous septum and left fibrous trigone, respectively, and both are continuous with the dorsal side of the right fibrous trigone. The aorta apparently is in a wedged position between the right and left atrioventricular anuli. The right fibrous trigone is also connected to the atrial myocardium but fingerlike protrusions of collagen are not present. In three specimens the noncoronary sinus distal to the right fibrous trigone was closely related to atrial muscle fiber. In the others loose connective tissue was present between these structures.

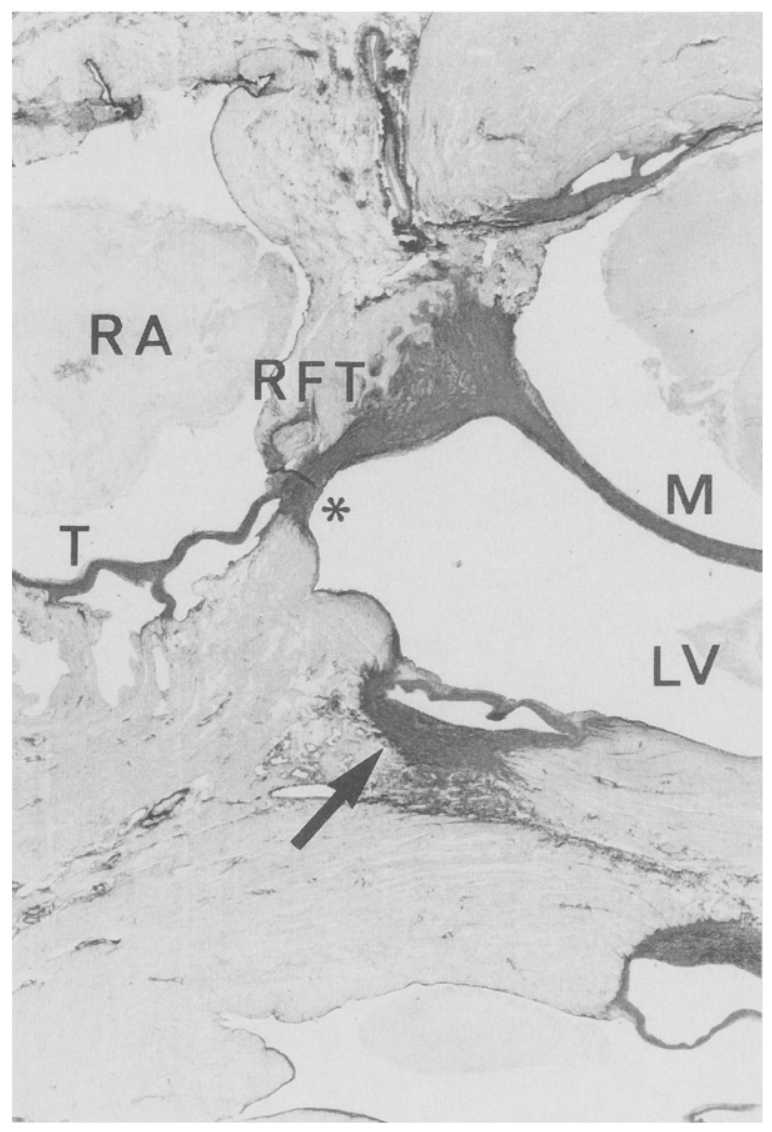

Fig. 7. Section of the proximal part of the aortic root. The collagen is darkly stained. The arrow indicates the "third body," situated at the proximal part of the right coronary sinus. The asterisk indicates the membranous septum. $R F T$, Right fibrous trigone; $R A$, right atrium; $M$, anterior mitral valve leaflet; $L V$, left ventricle; $T$, tricuspid valve leaflet.

Elastic lamella count. The site $2 \mathrm{~mm}$ distal to the commissures appeared to be representative for the distal part of the pulmonary trunk and aorta. The numbers of elastic lamellae at this level and in the middle of the sinus walls are presented in Table II. The difference between pulmonary trunk and aorta at the distal level was evident: there were more elastic lamellae in the aortic wall compared with the pulmonary wall (66 versus 52 ; $p<0.0008$ ). There was no statistically significant difference in the middle of the sinuses between the two arteries (40 and 39, respectively; $p=$ $0.46)$. Thus the difference within the aorta at the two levels $(p=0.000004)$ was larger than the difference within the pulmonary trunk $(p<$ 0.004). In the nine specimens studied, the two 
Table I. Proximal structures of the aortic root

\begin{tabular}{ll}
\hline \multicolumn{1}{c}{ Proximal structures } & \multicolumn{1}{c}{ Aortic circumference } \\
\hline Myocardium & Half left sinus \\
Left ventricular myocardium & Interleaflet triangle between left and right coronary sinuses \\
& Interleaflet triangle between left and right coronary sinuses \\
Septal myocardium & Right coronary sinus \\
Fibrous structures & Interleaflet triangle between right and noncoronary sinuses \\
Membranous septum & Noncoronary sinus \\
Right fibrous trigone & Interleaflet triangle between left and noncoronary sinuses \\
Anterior mitral valve leaflet & Left sinus \\
Left fibrous trigone &
\end{tabular}

adult hearts exhibited more elastic lamellae at the distal level of the two arteries than the other specimens. In the middle of the aortic and pulmonary sinus walls there were no differences.

\section{Discussion}

As recognized by several authors the components of the arterial roots comprise the vessel wall, characterized by concentric lamellae, ventricular structures (myocardial or collagenous), and a collagenous structure that is interposed between the vessel wall and the ventricular structures. ${ }^{1,2}$ It is this interposed collagenous structure, to which the valve leaflets are attached, that we consider to represent the anulus. Zimmerman ${ }^{16}$ in 1969 described this structure as "a crownlike formation of collagenous tissue." By 1923, Lewis and Grant ${ }^{1}$ had observed that the anulus "is not a simple ring." Anderson and colleagues $^{5}$ in 1991 opposed the use of the term aortic anulus on the grounds that "the attachments of the leaflets are not arranged in a ringlike fashion." Indeed, the leaflets follow a semilunar pattern. It is, however, not the attachment of the leaflets that we consider to represent the anulus, but the entire collagenous structure to which they are attached. This collagenous structure is basically a circular band that has its distal edge formed like a threepeaked crown, whereas its proximal border is characterized by three fimbriated, less pronounced curves. Its connection to the adjacent vessel wall distally and the ventricular structures proximally is very intricate. Notwithstanding the fact that in the case of the aorta a distinctive borderline cannot be seen between part of the anulus and the proximal fibrous structures, we consider the term anulus applicable.

The reversed relation of the elastic vessel wall and the collagenous anulus in the sinus wall (elastic layer inside) and in the interleaflet triangle (elastic layer
Table II. Elastic lamella count in nine heart specimens

\begin{tabular}{cll}
\hline & \multicolumn{2}{c}{ No. } \\
\cline { 2 - 3 } Level & Pulmonary trunk & \multicolumn{1}{c}{ Aorta } \\
\hline Sinuses & 39 (range 30-46) & 39 (range 34-45) \\
Children & $38(37$ and 39) & $42(40$ and 44) \\
Adults & & \\
Distal & $50($ range 42-65) & 63 (range 56-69) \\
Children & $59(55$ and 62) & $78(76$ and 79) \\
Adults &
\end{tabular}

outside) as found by us in both the pulmonary and aortic root had already been recognized in the aortic root by Lewis and Grant. ${ }^{1}$ The isolated condensations of elastic fibers in the interleaflet triangles of the aortic (more pronounced) and the pulmonary (less conspicuous) roots have, as far as we are aware, not been described before. They might be indicative of elastic properties to pass on end-diastolic left ventricular pressure, which is useful in the opening mechanism of the semilunar valves, as described for the aortic valve by Thubrikar and associates. ${ }^{17} \mathrm{~A}$ collagenous tendon between two opposing interleaflet triangles of the pulmonary and aortic roots was found in two of the nine specimens. This structure is known as the conus tendon. ${ }^{18,19}$ The prevalence of this tendon is not known. Kerr and Goss, ${ }^{20}$ having studied the relation between the pulmonary and aortic valves in 200 hearts, did not mention this tendon. The collagenous condensation at the proximal part of the right coronary sinus wall, as present in three of nine specimens, has been described before by Zimmerman, ${ }^{16,21}$ who assigned the term third body to it. He described it as being continuous with the anterior part of the membranous septum. This "third body" was, however, continuous with the membranous septum in only one of our specimens, whereas in the other two specimens the third body 
and the membranous septum were separated by muscular ventricular septum.

With respect to the differences between the pulmonary and aortic roots it appears that these are both major and minor. The anulus of the pulmonary root is anchored in myocardium over its entire circumference, whereas this applies to only half of the aortic circumference. The right ventricular myocardium is thin, thus providing a more delicate attachment of the pulmonary anulus. This contrasts with the thick left ventricular myocardium and the proximal continuation of more than half of the aortic anulus in fibrous structures such as the membranous septum, the right fibrous trigone, the anterior leaflet of the mitral valve, and the left fibrous trigone. The sinus walls and the interleaflet triangles of the aorta appear to be thicker. Surprisingly, we found no significant differences between the elastic lamella counts in the pulmonary and aortic sinuses, nor was there a significant difference at this level between the counts in children and adults. Although the number of studied specimens was small, it appears that the number of lamellae in the sinuses is fixed. The difference in thickness of the sinus walls might be the result of a difference in the amount of collagen, smooth muscle cells, or ground substance and/or in the thickness of the elastic lamellae themselves. At the level distal to the commissures, the number of elastic lamellae in both arteries was higher than that in the sinus walls. In contrast to the situation in the sinus walls, at the distal level, this number is not fixed. In agreement with the findings of others, ${ }^{22,23}$ the adult specimens showed a higher count. The aorta has more elastic lamellae than the pulmonary trunk and this difference was even more pronounced in the adult heart specimens. With regard to a possible growth potential of these lamellae no conclusions can be drawn, because the thickness of the lamellae, their organization, and other components of the medial layer of the vessel wall have to be taken into account. ${ }^{24,25}$

With regard to the surrounding structures the aorta is better encased. In addition to the thick left ventricular myocardium that bulges and forms a collar around the proximal part of the aortic root, the aortic root is wedged between the right and left atrioventricular anuli and atrial myocardium. The pulmonary root is only slightly supported by the thin right ventricular myocardium and the adjacent aorta.

When the pulmonary autograft is used for aortic root replacement the following aspects are relevant. It should be appreciated that the structures of the semilunar valve leaflets ${ }^{2}$ and the walls of the sinuses in both roots are not essentially different. The pulmonary autograft, however, has thinner interleaflet triangles, the proximal border consists of a ridge of relatively thin right ventricular myocardium, and it does not contain the proximal fibrous structures of the left ventricular outflow tract. For this reason the pulmonary autograft should be trimmed to leave only a few millimeters of right ventricular myocardium as suture area, followed by implantation at the level of the anulus ${ }^{9}$ to obtain maximal support of the fibrous structures of the left ventricular outflow tract and the surrounding ventricular and atrial myocardium. In contrast, in an extended autograft procedure this support is not optimal because these fibrous structures are not left intact as a result of the procedure.

We thank the Department of Pathology of the University Hospital Rotterdam for permission to study two of their heart specimens.

\section{REFERENCES}

1. Lewis T, Grant RT. Observations relating to subacute infective endocarditis. Heart 1923;10:21-99.

2. Gross L, Kugel MA. Topographic anatomy and histology of the valves in the human heart. Am J Pathol 1931;7:445-73.

3. Brewer RJ, Deck DJ, Capati B, Nolan SP. The dynamic aortic root: its role in aortic valve function. $\mathbf{J}$ Thorac Cardiovasc Surg 1976;72:413-7.

4. Angelini A, Ho SY, Anderson RH, et al. The morphology of the normal aortic valve as compared with the aortic valve having two leaflets. J Thorac Cardiovasc Surg 1989; 98:362-7.

5. Anderson RH, Devine WA, Ho SY, Smith A, McKay R. The myth of the aortic anulus: the anatomy of the subaortic outflow tract. Ann Thorac Surg 1991;52:640-6.

6. Ross DN. Aortic root replacement with a pulmonary autograft: current trends. J Heart Valve Dis 1994;3:358-60.

7. Ross DN. Replacement of aortic and mitral valves with a pulmonary autograft. Lancet 1967;2:956-8.

8. Matsuki O, Okita Y, Almeida RS, et al. Two decades' experience with aortic valve replacement with pulmonary autograft. J Thorac Cardiovasc Surg 1988;95:705-11.

9. Hokken RB, Bogers AJJC, Taams MA, et al. Aortic root replacement with a pulmonary autograft. Eur J Cardiothorac Surg 1995;9:378-83.

10. Kouchoukos NT, Davila-Roman VG, Spray TL, Morphy SF, Perillo JB. Replacement of the aortic root with a pulmonary autograft in children and young adults with aortic valve disease. N Engl J Med 1994;330:1-6.

11. Elkins RC, Knott-Craig CJ, Ward KE, McCue C, Lane MM. Pulmonary autograft in children: realized growth potential Ann Thorac Surg 1994;57:1387-94.

12. Pacifico AD, Kirklin JK, McGiffin DC, Matter GJ, Nanda NC, Diethelm AG. The Ross operation: early echocardio- 
graphic comparison of different operative techniques. J Heart Valve Dis 1994;3:365-70.

13. Bellhouse BJ, Bellhouse F, Abbott JA, Talbot L. Mechanism of valvular incompetence in aortic sinus dilatation. Cardiovasc Res 1986;34:83-94.

14. Roman MJ, Devereux RB, Niles NW, et al. Aortic root dilatation as a cause of isolated, severe aortic regurgitation. Ann Intern Med 1987;106:800-7.

15. Kirklin JW. Anatomy, dimensions, and terminology. In: Kirklin JW, Barratt-Boyes B, eds. Cardiac surgery. New York: John Wiley, 1993:3-60.

16. Zimmerman $\mathrm{J}$. The functional and surgical anatomy of the aortic valve. Isr J Med Sci 1969;5:862-6.

17. Thubrikar M, Nolan SP, Bosher LP, Deck JD. The cyclic changes and structure of the base of the aortic valve. Am Heart J 1980;99:217-24.

18. Patten BM. The development of the heart. In: Gould SE, ed. Pathology of the heart. Springfield, Illinois: Charles Thomas, 1960:24-92.
19. Tandler J. Anatomie des Herzens. Jena: Verlag von Gustav Fischer, 1913:140-51.

20. Kerr A, Goss CM. Retention of embryonic relationship of aortic and pulmonary valve cusps and a suggested nomenclature. Anat Rec 1956;125:777-82.

21. Zimmerman J. The functional and surgical anatomy of the heart. Ann R Coll Surg Engl 1966;39:348-66.

22. Plank L, James Y, Wagenvoort CA. Caliber and elastin content of the pulmonary trunk. Arch Pathol Lab Med 1980;104:238-41.

23. Wolinsky $\mathrm{H}$. Comparison of medial growth of the human thoracic and abdominal aortas. Cric Res 1970;27:531-8.

24. Schlatmann TJM, Becker AE. Histologic changes in the normal aging aorta: implications for dissecting aortic aneurysms. Am J Cardiol 1977;39:13-20.

25. Saldana M, Arias-Stefia J. Studies on the structure of the pulmonary trunk: 1-normal changes in the elastic configuration of the human pulmonary trunk at different ages. Circulation 1968;27:1086-93.

\section{Bound volumes available to subscribers}

Bound volumes of The Journal of Thoracic and Cardiovascular Surgery are available to subscribers (only) for the 1997 issues from the Publisher, at a cost of $\$ 110.50$ for domestic, $\$ 139.64$ for Canadian, and $\$ 130.50$ for international subscribers for Vol, 113 (January-June) and Vol. 114 (July-December). Shipping charges are included. Each bound volume contains a subject and author index and all advertising is removed. Copies are shipped within 60 days after publication of the last issue of the volume. The binding is durable buckram with the Journal name, volume number, and year stamped in gold on the spine. Payment must accompany all orders. Contact Mosby-Year Book, Inc., Subscription Services, 11830 Westline Industrial Drive, St. Louis, Missouri 63146-3318, USA; phone 800-453-4351 or 314-453-4351.

Subscriptions must be in force to qualify. Bound volumes are not available in place of a regular Journal subscription. 